Case Reports

\title{
Case Report and Review of Raoultella Planticola Urinary Tract Infection
}

\author{
${ }^{1}$ Christina M. Richards and ${ }^{2}$ Eric R. Musial \\ ${ }^{1}$ Pharmacy Practice, Massachusetts College of Pharmacy and Health Sciences, Boston, MA \\ ${ }^{2}$ Pharmacy Department, Lowell General Hospital, Lowell, MA, USA
}

\author{
Article history \\ Received: 20-07-2019 \\ Revised: $19-08-2019$ \\ Accepted: 31-08-2019 \\ Corresponding Author: \\ Eric R. Musial \\ Pharmacy Department, Lowell \\ General Hospital, Lowell, MA, \\ USA \\ Email: Eric.Musial@lowellgeneral.org
}

\begin{abstract}
This case report includes a brief review of Raoultella planticola infections in the literature and describes a case of a mildly immunocompromised patient who presents with signs and symptoms of urinary tract infection. Three days after hospitalization, the patient's urine sample had a culture speciation of Raoultella planticola. The infection was found to be resistant only to ampicillin and the patient was subsequently started on amoxicillin/clavulanate upon discharge. Largely thought to be nonpathogenic, R. planticola infection is now believed to be opportunistic and a discussion of risk factors in this patient is reviewed.
\end{abstract}

Keywords: Urinary Tract Infection, Anti Infective Agents, Immunocompromised Patient

\section{Introduction}

Raoultella planticola is an encapsulated gram-negative aerobic bacillus that is part of the enterobacteriacea family (Kalaria et al., 2017). It is a histamine-producing bacterium generally found in aquatic environments, in fish and in soil (Lam and Salit, 2017). It is not generally thought to be pathogenic, but recent cases of human infection are emerging, most commonly in fragile, frequently hospitalized patients with multiple comorbidities and in the pediatric population (Kalaria et al., 2017; Mehmood et al., 2018; Serkan et al., 2018; Skelton et al., 2017; Xu et al., 2016). There is one concerning case report of carbapenem resistance with $R$. planticola infection (Skelton et al., 2017).

$R$. planticola is closely related to Klebsiella and was originally classified as Klebsiella trevisanii after its discovery in 1984 and reclassified in 2001. (Mehmood et al., 2018) There have been 31 documented case reports of Raoultella planticola, four of which were cases of urinary tract infection. Other cases include reports of pneumonia, wound infection, cholangitis, bacteremia and conjunctivitis (Serkan et al., 2018). In this case, we present a patient with a confirmed case of Raoultella planticola urinary tract infection (Mehmood et al., 2018; Serkan et al., 2018).

\section{Case Presentation}

A 78 year old female with a 60 pack-year smoking history had a past medical history of lung cancer, status post resection for two separate lesions, Chronic Obstructive Pulmonary Disease (COPD), peripheral artery disease with abdominal aneurism stenting, hypertension, hyperlipidemia, gastroesophageal reflux disease and anxiety. She presented to the hospital via ambulance post fall and head strike. Medications included a Proton Pump Inhibitor (PPI) and oral corticosteroids.

Computerized axial tomography studies were ordered to determine the cause of her fall. Differential diagnosis included brain metastasis, stroke, or hemorrhage. It was noted on presentation that her temperature was $102.9^{\circ} \mathrm{F}$ and heart rate of 110 beats per minute. The patient had mild left lower quadrant pain and three episodes of vomiting. Her urinalysis was positive for blood, nitrites, leukocyte esterase, WBC count of 14/HPF and 4+ bacteria. Her complete blood count was remarkable for a WBC count of $18.8 \mathrm{~mm}^{3}, \mathrm{RBC}$ count of $3.61 \mathrm{Mil} / \mathrm{mm}^{3}$, $\mathrm{Hgb}$ of $11.6 \mathrm{Gm} / \mathrm{dL}$ and Hct of $34.4 \%$. There was evidence of a left shift, with an absolute neutrophil count of $17.62 \mathrm{~mm}^{3}$. Lactic acid was not elevated and other routine chemistries were unremarkable, but her troponin was elevated at $0.171 \mathrm{nGm} / \mathrm{mL}$ and her C-Reactive Protein was elevated to $5.24 \mathrm{mg} / \mathrm{dL}$.

As labs and vitals were concerning for urinary tract infection with possible sepsis, the patient received 1 gram of ceftriaxone and 4.5 grams of piperacillin/tazobactam in the emergency room. When transferred to the floor, she was started on empiric antibiotics including cefepime $1 \mathrm{~g}$ every 8 hours and vancomycin dosed by a trough of 15-20 $\mathrm{mg} / \mathrm{dL}$, as patient was immunocompromised with lung 
cancer and frequent admissions. Empiric antibiotics were continued for four days until culture results were received.

Three days after presentation, urine cultures were positive for Raoultella planticola, resistant to ampicillin and susceptible to amoxicillin/clavulanate, ampicillin/sulbactam, aztreonam, cefazolin, cefepime, ceftriaxone, ciprofloxacin, ertapenem, gentamicin, impenem, levofloxacin, meropenem, nitrofurantoin and sulfamethoxazole/trimethoprim. The patient was continued on cefepime until her planned discharge when she was transitioned to amoxicillin/clavulanate for a total of 10 days of antibiotics.

The patient's fever resolved by the end of her second day of hospitalization and she no longer complained of dysuria. Her urinary tract infection had resolved upon her discharge.

\section{Discussion}

Many risk factors predisposed this patient to an opportunistic infection. The patient was mildly immune suppressed from previously treated lung cancer, which during this admission was found to have metastasized to the brain. In addition, she suffered from COPD, for which she was frequently treated with oral corticosteroids that left her vulnerable to infection. In multiple case reports, PPIs were highly utilized in patients who were found to have Raoultella planticola infection. This patient was on a chronic PPI, which could have been a contributing factor to this infection, or as PPIs are commonly used, this could also be coincidence.

The laboratory equipment used for microbial identification and antibiotic susceptibilities was the Vitek 2.240 system manufactured by Biomerieux. This case is consistent with most other cultures and sensitivities reported in other case reports. It is possible that with other laboratory equipment, this bacterium could be absent in reporting, or could be reported as Klebsiella strains. Though not commonly speciated, as laboratory equipment continues to improve, more cases may emerge in the future.

Although both Klebsiella sp. and Raoultella planticola can both be classified as opportunistic in nature, Klebsiella is often healthcare acquired whereas $R$. planticola case reports describe patients coming in contact with a source out in the community, such as contaminated water and there are an absence of hospital acquired cases in the literature.

\section{Conclusion}

In conclusion, Raoultella planticola, a gram-negative bacterium that is generally non-pathogenic, is becoming more prevalent in immunocompromised patients. While generally nonresistant to most gram-negative antibiotic treatments, unlike Klebsiella, its resistance patterns are still developing.

\section{Ethics}

This article is original and contains unpublished material. The corresponding author confirms that all of the other authors have read and approved the manuscript and no ethical issues involved.

\section{References}

Kalaria, S.S., K. Elliott, N. Combs and L.G. Phillips, 2017. Raoultella planticola: A rare cause of wound infection. Wounds, 29: e103-e105.

Lam, W. and I. Salit, 2017. Raoultella planticola bacteremia following consumption of seafood. Canadian J. Infect. Dis. Med. Microbiol., 25: e83-e84. DOI: $10.1155 / 2014 / 439598$

Mehmood, H., N. Pervin, M. Israr ul haq, K.R. Kamal and A. Marwat et al., 2018. A rare case of raoultella planticola urinary tract infection in a patient with immunoglobulin a Nephropathy. J. Investig. Med. High Impact. Case Rep. DOI: $10.1177 / 2324709618780422$

Serkan, A., A.U. Zeynep, O.D. Sevliya, A. Gülşen and Y. Nurhayat et al., 2018. A rare and emerging pathogen: Raoultella planticola identificaion based on 16s rRNA in an infant. J. Infect. Public Health, 11: 130-132. DOI: 10.1016/j.jiph.2017.03.006

Skelton, W.P., Z. Taylor and J. Hsu, 2017. A rare case of Raoultella planticola urinary tract infection in an immunocompromised patient with multiple myeloma. IDCases, 8: 9-11. DOI: $10.1016 /$ j.idcr.2017.02.002

Xu, M., W. Xie, Y. Fu, H. Zhou and J. Zhou, 2016. Nosocomial pneumonia caused by carbapenemresistant Raoultella planticola: A case report and literature review. Infection, 43: 245-248. DOI: $10.1007 / \mathrm{s} 15010-015-0722-9$ 